\title{
Antioxidant, antimicrobial and neutrophil-modulating activities of herb extracts
}

\author{
Petko Denev ${ }^{1}$, Maria Kratchanova ${ }^{\boxplus}$, Milan $\mathrm{Ciz}^{2}$, Antonin Lojek², Ondrej Vasicek ${ }^{2}$, Denitsa \\ Blazheva ${ }^{3}$, Plamena Nedelcheva ${ }^{3}$, Libor Vojtek ${ }^{4}$ and Pavel Hyrs ${ }^{4}$
}

IInstitute of Organic Chemistry with Centre of Phytochemistry, Bulgarian Academy of Sciences, Laboratory of Biologicaly Active Substances, Plovdiv, Bulgaria; ${ }^{2}$ nnstitute of Biophysics of the AS CR, Brno, Czech Republic; ${ }^{3}$ University of Food Technologies, Plovdiv, Bulgaria; ${ }^{4}$ Department of Animal Physiology and Immunology, Institute of Experimental Biology, Masaryk University, Brno, Czech Republic

The present study provides a comprehensive data on the antioxidant, antimicrobial and neutrophil-modulating activities of extracts from six medicinal plants - blackberry (Rubus fruticosus) leaves, chokeberry (Aronia melanocarpa) leaves, hawthorn (Crataegus monogyna) leaves, lady's mantle (Alchemilla glabra) aerial parts, meadowsweet (Filipendula ulmaria) aerial parts and raspberry (Rubus idaeus) leaves. In order to analyze the antioxidant activity of the herbs, several methods (ORAC, TRAP, HORAC and inhibition of lipid peroxidation) were used. Blackberry leaves and meadowsweet extracts revealed the highest antioxidant activities via all methods. All extracts studied blocked almost completely the opsonized zymosan particle-activated ROS production by neutrophils from human whole blood. On the other hand, the effect of extracts on phorbol myristate acetate-activated ROS production was much milder and even nonsignificant in the case of chokeberry leaves. This latter result suggests that extracts (apart from their antioxidative activity) interfere with the signaling cascade of phagocyte activation upstream of the protein kinase $C$ activation. The antimicrobial activity of the investigated extracts against 11 human pathogens was investigated using three different methods. Meadowsweet and blackberry leaves extracts had the highest antimicrobial effect and the lowest minimal inhibiting concentrations (MICs) against the microorganisms tested.

Key words: herbs, polyphenols, antioxidant activity, antimicrobial activity, phagocytes, reactive oxygen species

Received: 31 January, 2014; revised: 15 April, 2014; accepted: 08 May, 2014; available on-line: 18 June, 2014

\section{INTRODUCTION}

Human gastrointestinal tract (GIT) is constantly exposed to oxidizing agents of diverse origins. The diet contains various prooxidants, including metals (copper and free or heme-bound iron), lipid hydroperoxides, aldehydes and nitrite, and consequently elevated levels of lipid peroxides have been observed in the postprandial state (Kanner \& Lapidot, 2001). These prooxidants may induce oxidative stress in the GIT, resulting in stomach ulcer and stomach, colon, and rectal cancers. Besides that, oxidative stress induced by microbial infections is another major contributor to the development of gastro-intestinal diseases. There is increasing evidence that microbial pathogens induce oxidative stress in host cells, resulting in considerable accumulation of inflammatory cell, which could be related to the development of gastric mucosal as well as neuromuscular disorders (Suzuki et al., 2012). Recent studies have shown that many bacterial pathogens can trigger apoptosis of infected host cells (Krzyminska et al., 2011). It has been shown that phagocyte-derived reactive oxygen species (ROS) and reactive nitrogen species (RNS) are important factors inducing apoptosis (Circu \& Aw, 2010). The activation of phagocytes in the gut may also increase the ROS and RNS production, and gastric juice may promote lipid peroxidation (Kanner \& Lapidot, 2001). Phagocytic cells such as macrophages and neutrophils play a key role in innate immunity owing to their ability to recognize, ingest, and destroy pathogens by oxidative and nonoxidative mechanisms. In response to a variety of stimuli, NADPH oxidase present in neutrophils is activated in a phenomenon described as the respiratory burst, characterized by the production of the superoxide anion which gives rise to other forms of ROS. In addition nitric oxide (NO) and other RNS produced mainly by macrophages are among the major microbicidal agents of inflammation during the fight against pathogenic microorganisms and tumor cells. However, excessive or inappropriate ROS and RNS production by phagocytes is associated with oxidative damage to membrane lipids, DNA, proteins, and lipoproteins, resulting in various autoimmune and inflammatory diseases. Thus, the modulation of inflammation and oxidative stress by natural substances can be beneficial. Therefore, the role of antioxidants in the GIT may be very important and antioxidants contained in foods could suppress the oxidative stress and related diseases in the gastrointestinal tract before being absorbed (Halliwell et al., 2000) and thus could modulate the level of oxidative stress by enhancing anti-inflammatory or antioxidant capacity (Suzuki et al., 2012). In the recent search for novel sources of anti-inflammatory and antioxidant agents medicinal plants have attracted particular attention. Since ancient times herbs have been used in

e-mail: Ibas@plov.omega.bg

Abbreviations: AAPH, 2,2'-azobis (2-amidino-propane) dihydrochloride; AUC, area under the curve; $\mathrm{CL}$, chemiluminescence; $\mathrm{DW}$, dry weight; GAE, gallic acid equivalents; GIT, gastrointestinal tract; HBSS, Hank's balanced salt solution; HORAC, hydroxyl radical averting capacity; HPLC, high performance liquid chromatography; LPS, lipopolysaccharide; MIC, minimal inhibiting concentration; ORAC, oxygen radical absorbance capacity; OZP, opsonized zymosan particles; PKC, protein kinase C; PLD, phospholipase D; PMA, phorbol myristate acetate; RLU, relative light units; RNS, reactive nitrogen species; ROS, reactive oxygen species; S.D., standard deviation; TE, trolox equivalents; TRAP, total peroxyl-radical antioxidant parameter 
numerous areas, including nutrition, healing, cosmetics, etc., without the exact mechanisms of their action being known. In our recent study we observed that herbs are a rich source of polyphenol compounds (Kratchanova et al., 2010) and their antioxidant activities are several times higher than those of vegetables (Ciz et al., 2010) and fruits (Denev et al., 2013). There was also a good correlation between the polyphenol content and the ORAC antioxidant activity of the herbs investigated, indicating that the polyphenol compounds are responsible for the free-radical scavenging capacity. Bearing in mind the great variety of medicinal plants and their potent antioxidant properties, herbs appear to be a valuable but still poorly-explored source of natural antioxidant and antimicrobial agents. Therefore, the aim of the present study was to investigate the polyphenol composition and content of six less-investigated herbs and to determine their antioxidant and antimicrobial activities, and their effect on the production of ROS by activated neutrophils. In order to obtain a broader view on their antioxidant properties we used several antioxidant activity assays (ORAC, TRAP, HORAC and inhibition of lipid peroxidation) relying on different mechanisms. The antimicrobial properties and the minimal inhibiting concentration of the herb extracts were investigated against 11 human pathogens and a genetically modified luminescent E. coli was used for the first time to determine the antimicrobial activity of natural products.

\section{MATERIALS AND METHODS}

Chemicals. Fluorescein disodium salt, 2,2-azobis-(2-amidino-propane)dihydrochloride (AAPH), 6-hydroxy-2,5,7,8-tetramethylchroman-2-carboxylic acid (trolox), $\mathrm{CoF}_{2} \times 4 \mathrm{H}_{2} \mathrm{O}$, picolinic acid, linoleic acid and HPLC reference compounds (gallic acid, 3,4-dihydroxybenzoic acid, chlorogenic acid, caffeic acid, p-coumaric acid, ferulic acid, ellagic acid, catechin, epicatechin, rutin, naringin, myrecetin, quercetin, naringenin and kaempherol) were purchased from Sigma-Aldrich (Steinheim, Germany). Folin-Ciocalteu's phenol reagent was purchased from Merck (Darmstadt, Germany) and luminol from Molecular Probes, (Eugene, Oregon, USA). All other reagents used were of analytical grade and purchased from local distributors.

Herb samples. All medicinal plants used were either obtained from local pharmacies (Plovdiv, Bulgaria) or collected from nature in 2012. The choice of the plants investigated was based on the available data on their biological activities. The following medicinal plants were investigated: blackberry (Rubus fruticosus) - leaves, chokeberry (Aronia melanocarpa) — leaves, hawthorn (Crataegus monogyna) - leaves, lady's mantle (Alchemilla glabra) - aerial parts, meadowsweet (Filipendula ulmaria) - aerial parts and raspberry (Rubus idaeus) - leaves. Freshly collected plants were dried in the shade at room temperature and all plant materials were inspected for contaminants. $1 \mathrm{~kg}$ of each herb was packed in paper bags and stored at ambient temperature prior to extraction and analysis.

Extraction. All plant materials were subjected to extraction under the following conditions: $50 \mathrm{~g}$ of the dried plant material was powdered in a laboratory mill. Then $5 \mathrm{~g}$ of the powder was transferred into extraction tubes and mixed with $100 \mathrm{ml}$ of the extragent $(80 \%$ acetone in $0.2 \%$ formic acid). Extraction was conducted on an orbital shaker at room temperature for one hour. After that, the samples were centrifuged $(6000 \times g)$ and super- natants collected. Extracts were concentrated via rotary evaporation to a volume of $15 \mathrm{ml}$ in order to remove the acetone. Then the volume was adjusted to $50 \mathrm{ml}$ with ultra clean water and the extracts were centrifuged. Clear supernatants were used for all further analysis after proper dilutions.

HPLC analysis of phenolic compounds. High Performance Liquid Chromatography (HPLC) analysis of phenolic components was performed on an Agilent 1220 HPLC system (Agilent Technology, Palo Alto, USA) equipped with a binary pump and UV-Vis detector (Agilent Technology) set at $280 \mathrm{~nm}$. TC-C18 column (5 $\mu \mathrm{m}, 4.6 \mathrm{~mm} \times 250 \mathrm{~mm}$ ) was used at $25^{\circ} \mathrm{C}$. The mobile phases were $0.5 \%$ acetic acid and $100 \%$ acetonitrile at a flow rate of $0.8 \mathrm{ml} / \mathrm{min}$. The eluent gradient started with $14 \%$ acetonitrile for $6 \mathrm{~min}$, and linearly increased to $25 \%$ acetonitrile in 24 minutes and to $50 \%$ acetonitrile in 10 min. Neochlorogenic acid content was calculated as chlorogenic acid equivalents. Results are given as mean values from three measurements of the same extract and expressed as mg per 100 grams of dry weight (DW).

Total polyphenol compounds analysis. Total polyphenols were determined according to the method of Singleton \& Rossi (1965) with Folin-Ciocalteu's reagent. Gallic acid was employed as a calibration standard and the results were calculated as mean values from three measurements of the same extract and expressed as gallic acid equivalents (GAE) per 100 grams of dry weight.

ORAC assay. ORAC was measured according to the method of Ou et al. (2001) with some modifications (Ciz et al., 2010). The method measures the antioxidant scavenging activity against peroxyl radical generated by thermal decomposition of 2,2'-azobis (2-amidino-propane) dihydrochloride (AAPH) at $37^{\circ} \mathrm{C}$. Fluorescein was used as the fluorescent probe. The loss of fluorescence of fluorescein was an indication of the extent of its oxidation through reaction with the peroxyl radical. The protective effect of an antioxidant was measured by assessing the fluorescence area under the curve (AUC) plot relative to that of a blank in which no antioxidant was present. Solutions of AAPH, fluorescein and trolox were prepared in a $\mathrm{Na}$ phosphate buffer $(75 \mathrm{mmol} / 1, \mathrm{pH} 7.4)$. Samples were diluted in the phosphate buffer as well. Reaction mixture (total volume $200 \mu \mathrm{l}$ ) contained fluorescein $\left(170 \mu \mathrm{l}\right.$, final concentration $\left.5.36 \times 10^{-8} \mathrm{~mol} / \mathrm{l}\right)$, AAPH (20 $\mu \mathrm{l}$, final concentration $51.51 \mathrm{mmol} / \mathrm{l}$ ), and sample - $10 \mu \mathrm{l}$. The fluorescein solution and sample were incubated at $37^{\circ} \mathrm{C}$ for $20 \mathrm{~min}$ directly in a microplate reader, and $\mathrm{AAPH}$ (dissolved in buffer at $37^{\circ} \mathrm{C}$ ) was added. The mixture was incubated for $30 \mathrm{~s}$ before the initial fluorescence was measured. After that, fluorescence readings were taken at the end of every cycle (1 min) after shaking. For the blank, $10 \mu \mathrm{l}$ of phosphate buffer was used instead of the extract. Each herb extract was analyzed in two runs with four replicates and results are presented as mean value \pm standard deviation from the eight values. The antioxidant activity is expressed in micromole trolox equivalents (TE) per gram of dry weight (DW). Trolox solutions $(3.125,6.25 ; 12.5 ; 25$ and $50 \mu \mathrm{mol} / \mathrm{l})$ were used for defining the standard curve.

TRAP assay. Luminol-enhanced chemiluminescence (CL) was used to follow the peroxyl radical reaction and the principle was described previously in Cizova and coworkers (2004). The CL signal is driven by the production of luminal-derived radicals from thermal decomposition of AAPH. The TRAP value is determined from the duration of the time period $\left(T_{\text {sample }}\right)$ during which the 
sample quenched the CL signal due to the present antioxidants. Trolox solution $(8.0 \mathrm{nM})$ was used as a reference inhibitor $\left(T_{\text {trolox }}\right)$ instead of the sample. The calculation of the TRAP value followed the equation:

TRAP $=2.0[$ trolox $] T_{\text {sample }} / f T_{\text {trolox }}$,

where 2.0 is the stoichiometric factor of trolox (the number of peroxyl radicals trapped per one molecule of trolox) and $f$ is the dilution of the sample. All herb extracts were analyzed in two runs with three replicates each, and results are presented as mean \pm standard deviation from all six values.

HORAC assay. The HORAC assay developed by $\mathrm{Ou}$ and coworkers (2002) measures the metal-chelating activity of antioxidants in the conditions of Fenton-like reactions employing a $\mathrm{Co}(\mathrm{II})$ complex and hence the protecting ability against formation of hydroxyl radical. Hydrogen peroxide solution of $0.55 \mathrm{M}$ was prepared in distilled water, and $4.6 \mathrm{mM} \mathrm{Co(II)} \mathrm{was} \mathrm{prepared} \mathrm{as} \mathrm{fol-}$ lows: $15.7 \mathrm{mg}$ of $\mathrm{CoF}_{2} \times 4 \mathrm{H}_{2} \mathrm{O}$ and $20 \mathrm{mg}$ of picolinic acid were dissolved in $20 \mathrm{ml}$ of distilled water. Fluorescein $(170 \mu \mathrm{l}$, final concentration $60 \mathrm{nM})$ and $10 \mu \mathrm{l}$ of sample were incubated at $37^{\circ} \mathrm{C}$ for $10 \mathrm{~min}$ directly in a FLUOstar plate reader, then $10 \mu \mathrm{l}$ of $\mathrm{H}_{2} \mathrm{O}_{2}$ (final concentration $27.5 \mathrm{mM}$ ) and $10 \mu \mathrm{l}$ of $\mathrm{Co}(\mathrm{II})$ (final concentration $230 \mu \mathrm{M}$ ) solutions were added. Initial fluorescence was measured immediately, then readings were taken every minute after shaking. For the blank sample, phosphate buffer $(75 \mathrm{mM}, \mathrm{pH}=7.4)$ was used. Gallic acid solutions $(100,200,600,800$ and $1000 \mu \mathrm{M}$ in phosphate buffer) were used for standard curve. The AUC values were calculated as they were for the ORAC assay. Each herb extract was analyzed in two runs with four replicates and results are presented as mean value \pm standard deviation from the eight values. ORAC and HORAC analyses were carried out using a FLUOstar OPTIMA plate reader (BMG Labtech, Germany) with excitation wavelength of $485 \mathrm{~nm}$ and emission wavelength of $520 \mathrm{~nm}$.

Inhibition of lipid peroxidation assay. A modified assay described by Anthon \& Barrett (2001) and Kikuzaki and coworkers (2002) was used. A mixture of $1 \mathrm{ml}$ sample, $1 \mathrm{ml}$ of $83.5 \mathrm{mM}$ linoleic acid in $99.5 \%$ ethanol,

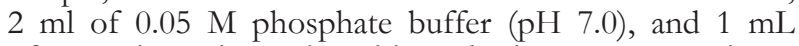
of water in a glass tube with a plastic stopper was incubated at $40^{\circ} \mathrm{C}$ in the dark. Absorbance at $234 \mathrm{~nm}$ was measured after one, three and six days of incubation on Infinite M200 microplate reader (Tecan, Austria). Initial values of absorbance (day zero before incubation) were subtracted from these values. For conversion of absorbance to the concentration of hydroperoxide, the extinction coefficient $23000 \mathrm{M}^{-1} \mathrm{~cm}^{-1}$ was used. Results are presented as mean values from three independent measurements each in duplicate.

Oxidative burst. Heparinized blood $(50 \mathrm{IU} / \mathrm{ml})$ from healthy volunteers $(n=7)$ was obtained by antecubital venipuncture. The oxidative burst of phagocytes in whole blood was measured chemiluminometrically using an LM-01 microplate luminometer (Immunotech, Czech Republic). The principle of the method was described previously (Ciz et al., 2007). Each reaction mixture consisted of $25 \mu \mathrm{l}$ of whole blood diluted $10 \times$ in Hank's balanced salt solution (HBSS) $\mathrm{pH} 7.4,2.5 \mu \mathrm{l}$ of plant extract, $25 \mu \mathrm{l}$ of $1 \mathrm{mM}$ luminol, and $25 \mu \mathrm{l}$ of one of the activators $62.5 \mu \mathrm{g} / \mathrm{ml}$ of opsonized zymosan particles (OZP; Sigma, USA) or $0.81 \mu \mathrm{g} / \mathrm{ml}$ of phorbol-12-myristate-13-acetate (PMA; Sigma, USA). HBSS was used to adjust the total reaction volume to $250 \mu \mathrm{l}$. The assays were run in duplicates. Measurements of spontaneous CL in samples containing blood and other substances except any activa- tor were included in each assay. The CL activity of the samples was measured immediately at $37^{\circ} \mathrm{C}$. Light emission expressed as relative light units (RLU) was recorded continuously for 60 minutes to obtain kinetic curves. Integrals of CL signal over $60 \mathrm{~min}$ were also calculated. Results are presented as means \pm standard error of the mean from six measurements.

Determination of antimicrobial activity by agar diffusion method. The agar diffusion method was used according to Denev and coworkers (2014). Eleven human pathogens (Escherichia coli ATCC 8739; Escherichia coli ATCC 25922; Salmonella sp.; Salmonella enterica ssp. enterica ATCC BAA-2162; Staphylococcus aureus ATCC 6538P; Staphylococcus aureus ATCC 25923; Listeria monocytogenes; Listeria monocytogenes I; Proteus vulgaris G; Pseudomonas aeruginosa ATCC 9027; Klebsiella pneumoniae) were used. All experiments were performed in duplicates and results are expressed as mean values.

Determination of minimal inhibiting concentration (MIC). The antimicrobial effects of the extracts were determined by analyzing the MIC using a 96 well microtiter plate method (broth dilution) according to the modified protocol described by Gutierrez and coworkers (2008). The first column of each plate contained 300 $\mu \mathrm{l}$ of nutrient broth (tryptone $-5.0 \mathrm{~g} / \mathrm{l}$, yeast extract $-2.5 \mathrm{~g} / \mathrm{l}$, glucose $-1 \mathrm{~g} / \mathrm{l}, \mathrm{pH} 7.0)$ and $100 \mu \mathrm{l}$ of the tested extract. From the second to the twelfth column, $200 \mu \mathrm{l}$ of broth were added and $200 \mu \mathrm{l}$ of the mixture from the first well was added to the second one and so on (serially diluted two-fold) along each row, until the eleventh column. The twelfth column was used for growth control (positive control) and a row was used for negative control at each dilution. Finally, $30 \mu \mathrm{l}$ of working culture of each test microorganism $\left(1 \times 10^{7} \mathrm{cfu} / \mathrm{ml}\right)$ was added to all the wells except the negative controls. The positive control contained distilled water instead of extract. Plates were incubated at $37^{\circ} \mathrm{C}$ for $24 \mathrm{~h}$ and then turbidity was determined at $600 \mathrm{~nm}$ in a microplate spectrophotometer (SPECTROstar ${ }^{\text {Nano }}$, BMG Labtech, Germany). The lowest concentration of the tested extract required to reduce the growth of the tested microorganism to $50 \%$ of the positive control was designated as the MIC. Results are presented as mean values from three independent measurements.

Antimicrobial activity against genetically modified Escherichia coli. In the following experiments transformed E. coli K12 resistant to ampicillin carrying the luxABCDEamp gene was used, expressing bacterial luciferase and its substrate (Atosuo et al., 2013). Bacteria were cultivated in LB medium with $100 \mu \mathrm{g}$ / $\mathrm{ml}$ ampicillin, washed twice in phosphate buffer $(\mathrm{pH}$ $7.0,133 \mathrm{mM}$ ) and adjusted with the phosphate buffer to the final concentration of approximately 330000 cells per $100 \mu \mathrm{l}$ before each measurement. The bioluminescence signal of bacterial suspensions after exposition to herb extracts was measured in relative light units (RLU) for one hour on LM-01T plate luminometer (Immunotech, Czech Republic) at $37^{\circ} \mathrm{C}$. The wells of microplates contained $180 \mu \mathrm{l}$ of bacterial suspension and $10 \mu \mathrm{l}$ (5\% concentration) of extracts, buffer was used as a control. The light emission during reaction is positively correlated with bacteria viability (Atosuo et al., 2013). To determine antibacterial properties of extracts, integrals (RLU*s) under kinetics curves were calculated and expressed as percentage of control. All samples were prepared in two independent repetitions and each repetition was analysed three times. Results are presented as mean values from the six measurements. 


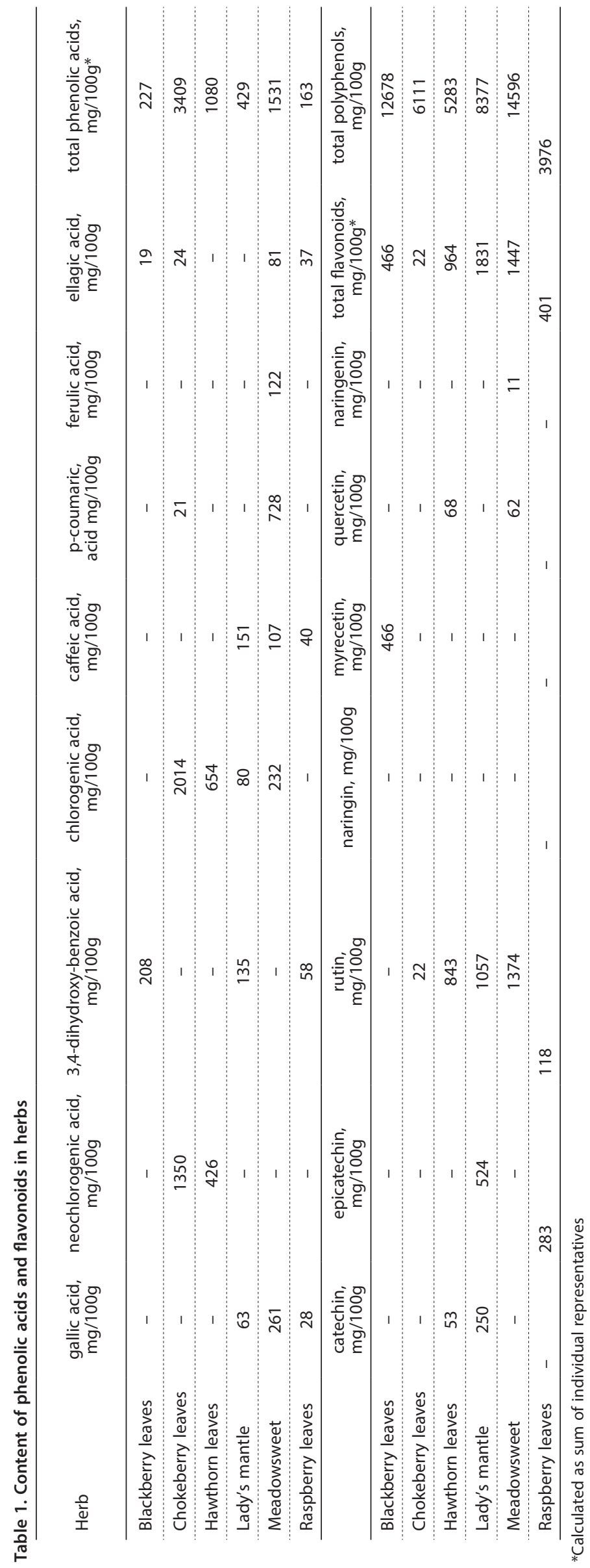

\section{RESULTS AND DISCUSSION}

\section{Polyphenol composition and antioxidant activity of herb extracts}

Results of the HPLC analysis of herb extracts are shown in Table 1 . The analyzed extracts showed substantial differences in their polyphenol composition and content. Aronia leaves are distinctive with a high content of hydroxycinnamic acids (chlorogenic and neochlorogenic) among polyphenol acids with a total content of $3409 \mathrm{mg} / 100 \mathrm{~g}$. Lady's mantle contains 3,4-hydroxybenzoic acid, chlorogenic acid and p-coumaric acid totaling $429 \mathrm{mg} / 100$ g. Among the investigated herbs, lady's mantle is the richest source of flavonoids, containing catechin (250 mg/100 g), epicatechin (524 $\mathrm{mg} / 100 \mathrm{~g}$ ) and a significant amount of rutin $(1057 \mathrm{mg} / 100 \mathrm{~g})$. The meadowsweet has the highest content of total plyphenols (14596.2 $\mathrm{mg} / 100 \mathrm{~g}$ ) and is a balanced source of phenolic acids (total content - $1531 \mathrm{mg} / 100 \mathrm{~g}$ ) and flavonoids - $1447 \mathrm{mg} / 100 \mathrm{~g}$. Hawthorn leaves contain $1080 \mathrm{mg} / 100 \mathrm{~g}$ of phenolic acids and $1831 \mathrm{mg} / 100 \mathrm{~g}$ of flavonoids. Blackberry leaves are a rich source of polyphenol compounds - $12678 \mathrm{mg} / 100$ g. They contain 3,4-dihydroxybenzoic acid (208 mg/100 $\mathrm{g})$, ellagic acid $(19 \mathrm{mg} / 100 \mathrm{~g})$ and myrecetin (466 mg/100 g). Raspberry leaves contain gallic, 3,4-dihydroxybenzoic, caffeic and ellagic acids. Epicatechin and rutin are the most abundant flavonoids in that herb. It is obvious from the results in Table 1 that the sum of the investigated phenolic acids and flavonoid contents is less than the total polyphenol content of the investigated herbs. We analyzed 16 of the most common polyphenol constituents in plants but it could be expected that other phenolic acids and flavonoids, not analyzed in this work were also present. Smolyakova et al. (2012) reported that lady's mantle polyphenol content is up to $9.6 \%$ of the dry herb weight, which is closed to our result $-8.4 \%$, but luteolin and apigenin, and their glycosides not analyzed in our study are the main flavonoids in that herb. Besides flavonoids and phenolic acids, medicinal plants are known to be a rich source of tannins (condensed and hydrolysable), which were not analyzed in the current study either. For example, Duckstein and coworkers (2013) identified 24 phenolic compounds in lady's mantle including pedunculagin and agrimoniin, and other monomeric and oligomeric ellagitannins, which constituted the major phenolic fraction. Gudej and Tomczyk (2004) reported that tannins are the main polyphenol constituents in raspberry and blackberry leaves. Fecka (2009) found 27 polyphenol compounds in meadowsweet, including substantial amounts of ellagitannins (up to $12500 \mathrm{mg} / 100 \mathrm{~g} \mathrm{DW}$ ) and rugosins A, B and $\mathrm{E}$ were identified for the first time as meadowsweet constituents. According to Fecka, the total polyphenol content of meadowsweet reached $16568 \mathrm{mg} / 100 \mathrm{~g}$, which is similar to our result — $14596 \mathrm{mg} / 100 \mathrm{~g}$. The high con- 
Table 2. ORAC, TRAP and HORAC antioxidant activity of herbs

\begin{tabular}{lccc}
\hline & $\begin{array}{c}\text { ORAC, } \\
\mu \mathrm{mol} \text { TE/g }\end{array}$ & $\begin{array}{c}\text { TRAP, } \\
\mu \mathrm{mol} \text { TE/g }\end{array}$ & $\begin{array}{c}\text { HORAC, } \\
\mu \mathrm{mol} \mathrm{GAE} / \mathrm{g}\end{array}$ \\
\hline Blackberry leaves & $1806 \pm 105$ & $2937 \pm 139$ & $2076 \pm 113$ \\
\hdashline Chokeberry leaves & $1363 \pm 69$ & $1263 \pm 56$ & $1120 \pm 53$ \\
\hline Hawthorn leaves & $1405 \pm 66$ & $1301 \pm 27$ & $882 \pm 203$ \\
\hline Lady's mantle & $1337 \pm 68$ & $1815 \pm 38$ & $1999 \pm 70$ \\
\hline Meadowsweet & $1555 \pm 85$ & $1993 \pm 102$ & $1640 \pm 223$ \\
\hline Raspberry leaves & $1349 \pm 119$ & $647 \pm 50$ & $1257 \pm 82$ \\
\hline
\end{tabular}

tent of hydrolysable tannins in that herb was confirmed by other studies (Barros et al., 2013). Chokeberry leaves' polyphenols have not been sufficiently investigated and data regarding their polyphenol composition and content are scarce. A recent study found 12 polyphenol compounds in chokeberry leaves and confirmed our results that chlorogenic and neochlorogenic acids are the main phenolic constituents (Lee et al., 2014). Beside that, dicaffeoylquinic acid was detected in high amounts, along with other minor components. Bearing in mind that chokeberry fruits contain substantial quantity of condensed tannins (Denev et al., 2012), we could expect that their leaves also accumulates significant amounts of these compounds.

The differences in the polyphenol content and composition of the investigated herbs are reflected in their biological activities and particularly antioxidant activity. When investigating antioxidant properties of natural antioxidants it is recommended to use more than one antioxidant assay for better understanding the principles of their antioxidant action (Ciz et al., 2010). Therefore, we used several assays addressing various aspects of the antioxidant action of polyphenols in our study ORAC, TRAP, HORAC and inhibition of lipid peroxidation. The methods used embrace different aspects of the antioxidant action and give a broader view on the antioxidant potential of the herb extracts. The ORAC and TRAP methods measure the ability of antioxidants to scavenge peroxyl radicals via hydrogen atom transfer. These radicals are physiologically the most important ones and the hydrogen atom transfer is the most physiologically relevant mechanism of antioxidant action. The HORAC method measures the metal-chelating activity of antioxidants under conditions of Fenton-like reactions and hence indicates the protecting ability against formation of hydroxyl radical. Results for the ORAC, TRAP and HORAC antioxidant activities are shown in $\mathrm{Ta}$ ble 2. In our previous study, we reported ORAC antioxidant activity of 25 herbs used traditionally in the Bulgarian folk medicine (Kratchanova et al., 2010). Compared to those results all the herbs investigated in the current study showed relatively high ORAC antioxidant activities, ranking among top ten herbs with the highest ORAC values. Blackberry leaves revealed the highest antioxidant activity using the three methods (ORAC - $1805.8 \mu \mathrm{mol}$ TE/g; TRAP - $2936.6 \mu \mathrm{mol} \mathrm{TE} / \mathrm{g}$; and HORAC 2075.6 $\mu \mathrm{mol}$ GAE/g). Meadowsweet showed the second highest ORAC and TRAP values - $1555.0 \mu \mathrm{mol} \mathrm{TE} / \mathrm{g}$ and $1992.7 \mu \mathrm{mol} \mathrm{TE} / \mathrm{g}$, respectively, indicating that this herb is a rich source of chain-braking polyphenolic antioxidants. Interestingly, lady's mantle extract revealed the second highest chelating ability expressed as a HORAC value of $1999.4 \mu \mathrm{mol} \mathrm{GAE} / \mathrm{g}$.

The effect of the extracts on lipid peroxidation was evaluated by assaying the autoxidation of linoleic acid
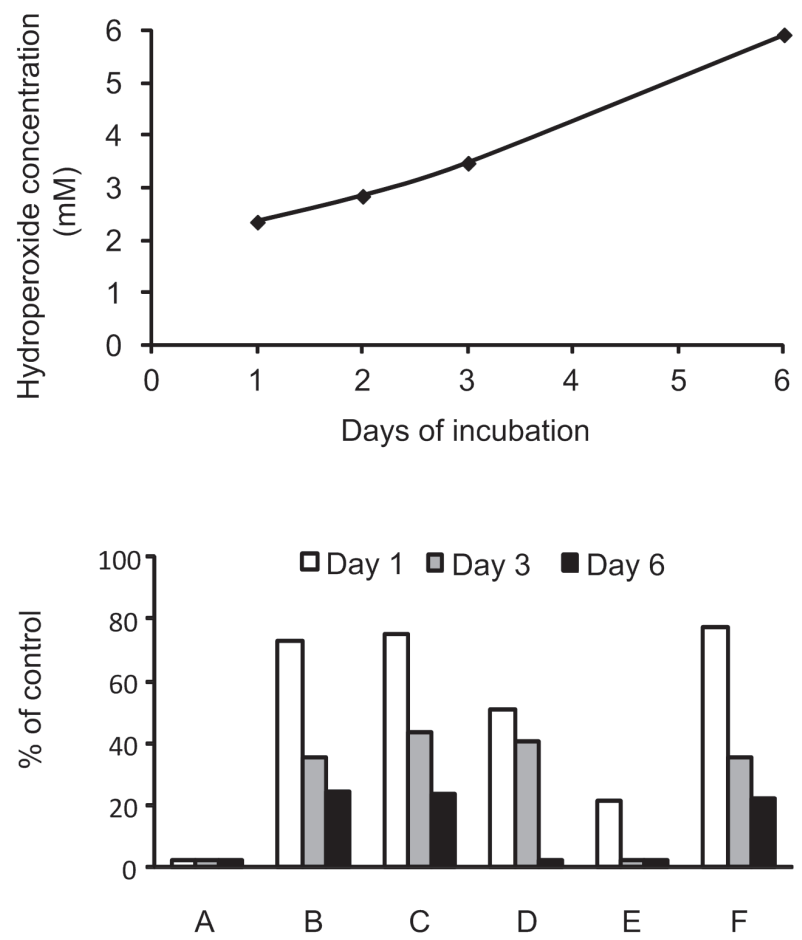

Figure 1. Inhibition of lipid peroxidation by herb extracts. Kinetics of hydroperoxide concentration in control samples without herb extracts during six days of autoxidation of linoleic acid (upper panel). Eeffect of herb extracts on hydroperoxide accumulation at the end of the $11^{\text {st }}, 3^{\text {rd }}$ and $6^{\text {th }}$ days of autoxidation of linoleic acid (lower panel). Results are expressed as percentage of control. A - blackberry leaves; B - chokeberry leaves; C - hawthorn leaves; D - lady's mantle; E - meadowsweet; $F$ - raspberry leaves.

in ethanol-buffer system by measuring the formation of linoleic acid hydroperoxides. This is a simple and reliable method for evaluation of the effects of potential antioxidants. As shown in Fig. 1A, the concentration of hydroperoxides rises gradually during the six days of linoleic acid autoxidation at $40^{\circ} \mathrm{C}$. The formation of hydroperoxides in the presence of the herb extracts is shown in Fig. 1B. It is obvious that the autoxidation of linoleic acid was very effectively inhibited, especially by the extract from blackberry leaves. In this case, the concentration of hydropexides was lowered to $2 \%$ of the control of all time points. The second most effective extract was that from meadowsweet. It inhibited the generation of hydroperoxides to $22 \%$ of control at day 1 and to $2 \%$ at days 3 and 6 . The third most effective was the extract from lady's mantle, followed by extracts from chokeberry, hawthorn and raspberry leaves. For the latter four extracts the inhibition of lipid peroxidation increased from day 1 to day 6 .

\section{Effect of herb extracts on ROS production by phagocytes}

The effects of studied extracts on the production of ROS by human whole blood phagocytes were studied using luminol-enhanced CL. The results are shown in Fig. 2 and expressed as kinetic curves of the CL response after OZP activation (upper panel), PMA activation (lower panel) and the integral values of the CL response. It is obvious that all the extracts studied significantly inhibited the ROS production, to the background value in the case of OZP-activated phagocytes. On the 

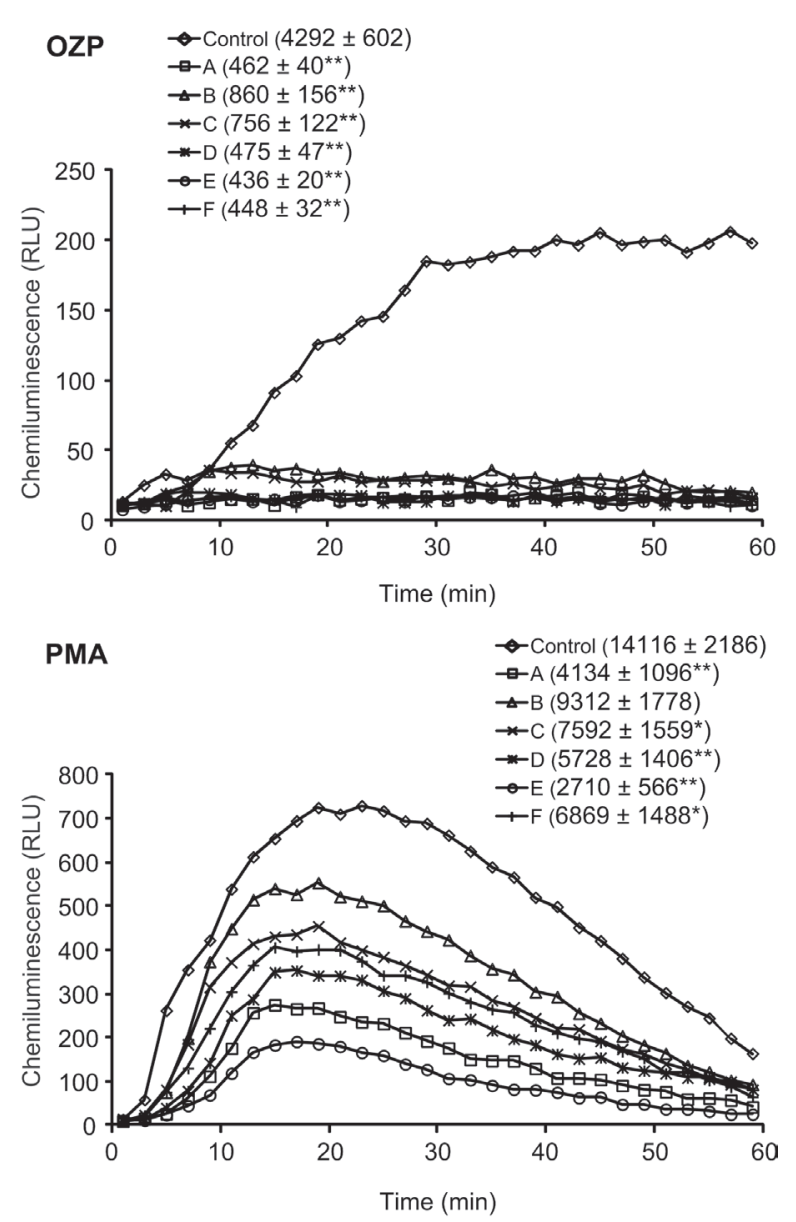

Figure 2. Effect of herb extracts on the production of reactive oxygen species by phagocytes.

Kinetic curves of the CL response after OZP activation (upper panel) and PMA activation (lower panel) are shown. A - blackberry leaves; B - chokeberry leaves; C - hawthorn leaves; D - lady's mantle; $E$ - meadowsweet; $F$ - raspberry leaves. Integral values of $\mathrm{CL}$ response (mean \pm S.E.M.) are also included. Statistically significant differences against control are shown by ${ }^{* *} p<0.01$ and ${ }^{*} p<0.05$.

other hand, some differences were observed when the effects were studied using PMA-activated human whole blood phagocytes. The most effective was the extract of meadowsweet $(p \leq 0.01)$ followed by blackberry leaves $(p \leq 0.01)$, lady's mantle $(p \leq 0.01)$, raspberry leaves $(p \leq 0.05)$, hawthorn leaves $(p \leq 0.05)$, chokeberry leaves. The least effective extract of chokeberry leaves still inhibited the PMA-activated CL response, but the effect was statistically non-significant. The inhibitory effects of the herb extracts on the PMA-activated CL response of phagocytes and partially also on the OZP-activated CL response of phagocytes could be caused by a direct ROS scavenging activity of the extracts due to their total polyphenol content. There was a strong negative correlation between the PMA-activated CL response and the total polyphenol content and a strong positive correlation between the TRAP antioxidative parameter and total polyphenols (not shown).

The effects of polyphenols as the basic constituents of various plant extracts on the respiratory burst of neutrophils have already been demonstrated. For example, cocoa flavonoids have been found to moderate a subset of signaling pathways derived from LPS stimulation of neutrophils, mainly the neutrophil oxidative burst and activation markers (Kenny et al., 2009). Those authors hypothesized that flavonoids could decrease the impact of LPS on the fMLP-primed neutrophil ability to generate ROS by partially interfering in the activation of the MAPK pathway. In another study, Ginkgo biloba extract containing flavonoids slowed down the respiratory burst of stimulated human neutrophils (Pincemail et al., 1987). An inhibitory effect of pure flavonoids on the respiratory burst of neutrophils has also been demonstrated (Pagonis et al., 1986; Zielinska et al., 2000). Wang and coworkers (2002) observed that cirsimaritin inhibited superoxide anion radical generation and oxygen consumption of neutrophils. On the other hand, cirsimaritin slightly enhanced the superoxide anion radical generation by PMA-activated NADPH oxidase. The results of those authors indicate that it is likely that the inhibition of the fMLP-induced respiratory burst by cirsimaritin in rat neutrophils is mainly through the blockade of the phospholipase D (PLD) signaling pathway. Selloum and coworkers (2001) observed that myricetin, quercetin, kaempferol and rutin inhibited the pholasin luminescence of fMLP-stimulated neutrophils. Lee and coworkers (2010) revealed that luteolin attenuated neutrophil respiratory burst but had a negligible effect on superoxide anion generation during PMA stimulation. Furthermore, luteolin effectively blocked MAPK/ERK kinase $1 / 2$ and Akt phosphorylation in fMLP-stimulated neutrophils. Thus, an inhibition of enzymes involved in signaling, rather than scavenging of superoxide anion radicals dominated in fMLP-stimulated neutrophils exposed to flavonoids in those studies. fMLP used in the cited studies is a soluble activator of neutrophils which binds to specific membrane receptors. Similarly, OZP binds to surface opsonin (either complement or Fc) receptors on phagocytes and triggers a signaling cascade leading finally to the activation of protein kinase $\mathrm{C}$ (PKC) with subsequent activation of NADPH oxidase, producing the superoxide anion - the first of the ROS produced by phagocytes. In contrast, PMA activates directly PKC, the step before NADPH oxidase activation. Our present results indicate in accordance with the literature that the herb extracts studied do not inhibit PKC or NADPH oxidase itself, but interfere with the signaling cascade of phagocyte activation upstream of the PKC activation.

\section{Antimicrobial activity of herbs}

The investigated plants are used in traditional medicine in the treatment of different infections (Shmid \& Gorris, 2007), but their antimicrobial properties are poorly investigated. Only few studies on antimicrobial activities of their extracts have been published to date. Gniewosz and coworkers (2014) determined the influence of meadowsweet flower extract on three pathogens and four saprophytes of apples, using fruit covered with a pullulan film containing the extract. Martini and coworkers (2009) analyzed the activity of blackberry leaves extract against Helicobacter pylori. In our study initial screening of the antimicrobial activity of the tested extracts was performed against eleven foodborne pathogens using the agar diffusion method. The antimicrobial activity was classified according to Rota and coworkers (2008) as weak (inhibition zone $<12 \mathrm{~mm}$ ), medium (inhibition zone between 12 and $20 \mathrm{~mm}$ ), and strong (inhibition zone above $20 \mathrm{~mm}$ ). The results are presented in Table 3. The blackberry and raspberry leaves extracts exhibited antimicrobial activity against all test microorganisms. In some cases single colonies were present in the inhibition zones (marked with an asterisk in Table $3)$. Some of the cells of E. coli ATCC 25922, both Sal- 


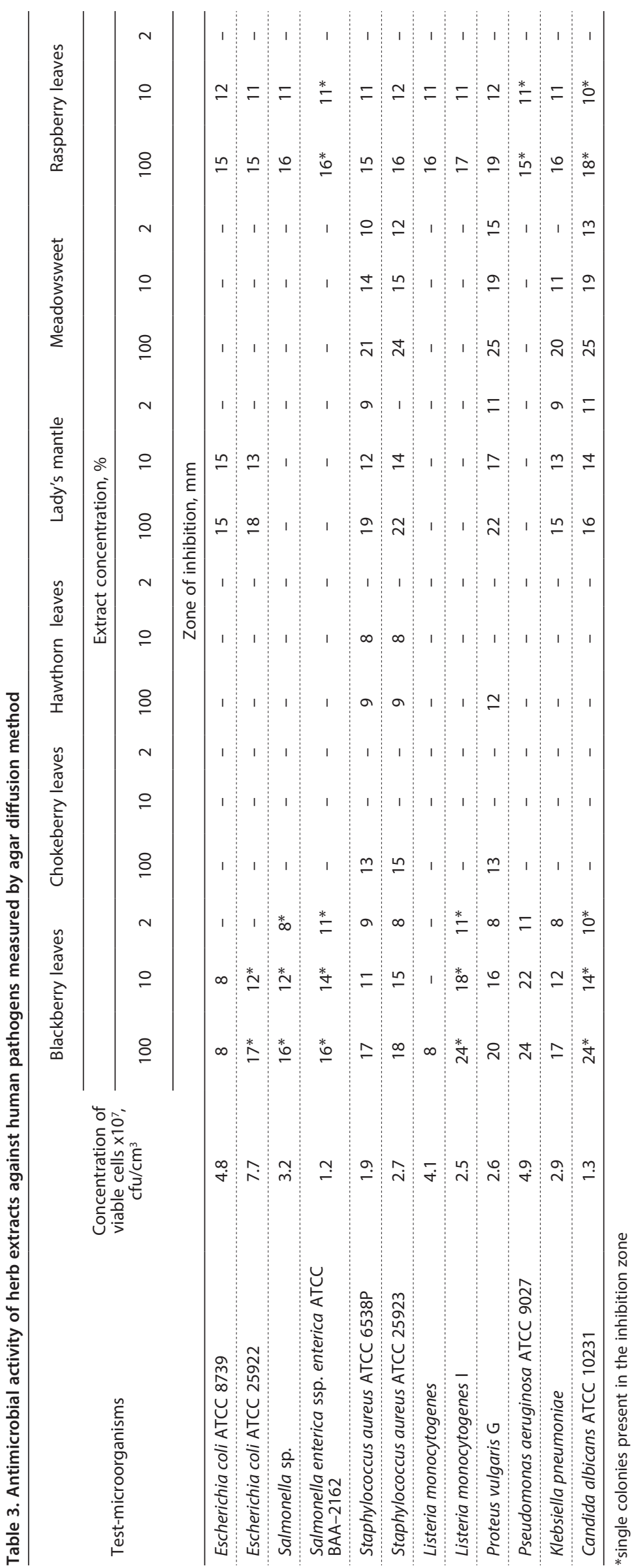

monella strains, L. monocytogenes I and C. albicans were less susceptible to the effect of the blackberry leaves extract and were able to grow in its presence. A number of $S$. enterica ssp. enterica ATCC BAA2162, P. aeruginosa and $C$. albicans cells were also more resistant to the activity of the raspberry leaves extract than others. The highest activity of the blackberry extract was observed against $P$. aeruginosa and $P$. vulgaris, where inhibition was detected even for $2 \%$ extract. The raspberry leaves extract produced the largest inhibition zones $(19 \mathrm{~mm})$ against $P$. vulgaris. Chokeberry and hawthorn leave extracts had medium and weak antimicrobial activites, respectively, against three of the test strains - the two $S$. aureus strains and $P$. vulgaris. The activity was observed only for undiluted extracts. Lady's mantle extract did not inhibit the growth of Salmonella, L. monocytogenes or $P$. aeroginosa, but antimicrobial activity against $S$. aureus ATCC $6538 \mathrm{P}$, P. vulgaris, K. pneumoniae and $C$. albicans was detected even when the lowest extract concentration was used. The activity against $S$. aureus ATCC 25923 and E. coli was weaker an inhibitory effect was observed only for $10 \%$ solutions. Meadowsweet extract also demonstrated antimicrobial activity against $S$. aureus strains, $P$. vulgaris, $K$. pneumoniae and C. albicans even in a $2 \%$ solution. Based on the obtained results four extracts (blackberry, lady's mantle, meadowsweet and raspberry leaves) were selected for further experiments.

The minimal inhibitory concentration $\left(\mathrm{MIC}_{50}\right)$ of the extracts was determined in order to quantify their antimicrobial activity (Table 4). The lowest $\mathrm{MIC}_{50}$ values were determined for the raspberry leaves extract against $S$. aureus ATCC $25923-0.031 \%$ and the meadowsweet extract against $P$. vulgaris and $K$. pneumoniae $-0.08 \%$. S. aureus ATCC 25923 was the most susceptible to the activity of the herb extracts. Its growth was inhibited at concentrations lower than $0.3 \%$ of the extracts in the medium. $\mathrm{MIC}_{50}$ values below $1 \%$ were found for: blackberry leaves against Salmonellas sp., $S$. aureus ATCC 6538P, P. vulgaris and $K$. pneumoniae; Lady's mantle against $P$. vulgaris; meadowsweet against $P$. vulgaris and $K$. pneumoniae; raspberry leaves against Salmonella sp. and Proteus vulgaris. For lady's mantle and raspberry leaves extracts the MICs were $2.5 \%$ against $S$. aureus ATCC 6538P. None of the extracts reduced the growth of the L. monocytogenes strains below 50\% of their initial viable cell counts. They also had low $\left(\mathrm{MIC}_{50}=25 \%\right)$ or no activity against $S$. enterica ssp. enterica ATCC BAA-2162, P. aeruginosa or E. coli strains.

All six extracts showed an activity against genetically modified E. coli. The bioluminescence signal decreased within few minutes after exposition of the bac- 
Table 4. Minimal inhibiting concentration $\left(\mathrm{MIC}_{50}\right.$ ) of chosen herb extracts against human pathogens

\begin{tabular}{|c|c|c|c|c|}
\hline \multirow{2}{*}{ Test microorganisms } & Blackberry leaves & Lady's mantle & Meadowsweet & Raspberry leaves \\
\hline & \multicolumn{4}{|c|}{$\mathrm{MIC}_{50,} \%$} \\
\hline Escherichia coli ATCC 8739 & 25 & - & 25 & - \\
\hline Escherichia coli ATCC 25922 & - & - & 25 & - \\
\hline Salmonella sp. & 0.19 & - & - & 0.78 \\
\hline Salmonella enterica ssp. enterica ATCC BAA-2162 & 25 & - & - & - \\
\hline Staphylococcus aureus ATCC 6538P & 0.63 & 2.5 & 0.63 & 2.5 \\
\hline Staphylococcus aureus ATCC 25923 & 0.13 & 0.25 & 0.25 & 0.031 \\
\hline Listeria monocytogenes & - & - & - & - \\
\hline Listeria monocytogenes I & - & - & - & - \\
\hline Proteus vulgaris $\mathrm{G}$ & 0.16 & 0.1 & 0.08 & 0.78 \\
\hline Pseudomonas aeruginosa ATCC 9027 & 25 & - & - & - \\
\hline Klebsiella pneumoniae & 0.63 & 25 & 0.08 & 25 \\
\hline
\end{tabular}

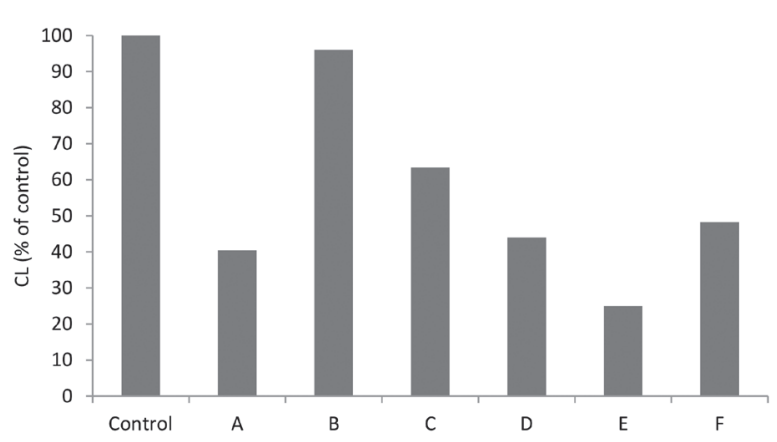

Figure 3. Luminometric measurement of antibacterial effect of herb extracts on E. coli viability.

Results are expressed as percentage of control samples without herb extracts. A - blackberry leaves; B - chokeberry leaves; $C$ — hawthorn leaves; D - lady's mantle; E - meadowsweet; F raspberry leaves.

teria to herb extracts and stayed low during the entire experiment. Meadowsweet showed the highest antibacterial activity (75\% inhibition of control, Fig. 3) followed by blackberry leaves (60\%), lady's mantle (56\%), raspberry leaves (52\%) and hawthorn leaves (27\%). A very low activity was found for chokeberry leaves with only $4 \%$ growth inhibition. The antibacterial activity of all the extracts supports the lipid peroxidation inhibition results and those regarding production of ROS by phagocytes.

\section{CONCLUSION}

Results obtained in the present study indicate that all herbs studied, especially blackberry leaves and meadowsweet, are rich sources of chain-breaking, chelating and lipid peroxidation inhibiting antioxidants, rendering high antioxidant activity measured by several methods. Besides that the herb extracts could prevent oxidative stress in the gastrointestinal tract trough modulation of neutrophils and an antimicrobial effect against a broad spectrum of human pathogens.

\section{Acknowledgement}

This work was funded by project № BG161PO0031.1.05-0024-C0001 "Development of nutraceuticals with antioxidant and immune-stimulating action" uder Operation 1.1.1: „Support for establishment and development of start-up innovative enterprizes" of Operational Program "Competitiveness" of the EU.

\section{REFERENCES}

Anthon GE, Barrett DM (2001) Colorimetric method for the determination of lipoxygenase activity. J Agr Food Chem 49: 32-37.

Atosuo J, Lehtinen J, Vojtek L, Lilius EM (2013) Escherichia coli K-12 (pEGFPluxABCDEamp): a tool for analysis of bacterial killing by antibacterial agents and human complement activities on a real-time basis. Luminescence 28: 771-779.

Barros L, Alves CT, Duenas M, Silva S, Oliveira R, Carvalho AM, Henriques M, Santos-Buelga C, Ferreira ICFR (2013) Characterization of phenolic compounds in wild medicinal flowers from Portugal by HPLC-DAD-ESI/MS and evaluation of antifungal properties. Ind Crop Prod 44: 104-110.

Circu M, Aw TY (2010) Reactive oxygen species, cellular redox systems, and apoptosis. Free Radical Bio Med 48: 749-762.

Ciz M, Cizova H, Denev P, Kratchanova M, Slavov A, Lojek A (2010) Different methods for control and comparison of the antioxidant properties of vegetables. Food Control 21: 518-523.

Ciz M, Komrskova D, Pracharova L, Okenkova K, Cizova H, Moravcova A, Jancinosva V, Petrikova M, Lojek A, Nosal R (2007) Serotonin modulates the oxidative burst of human phagocytes via various mechanisms. Platelets 18: 583-590.

Cizova H, Lojek A, Kubala L, Ciz M (2004) The effect of intestinal ischemia duration on changes in plasma antioxidant defence status in rats. Physiol Res 53: 523-531.

Denev P, Kratchanov Ch, Ciz M, Lojek A, Kratchanova M (2012) Bioavailability and antioxidant activity of black chokeberry (Aronia melanocarpa) polyphenols: in vitro and in vivo evidences and possible mechanisms of action. A review. Compr Rev Food Sci F 11: 471-489.

Denev P, Kratchanova M, Ciz M, Lojek A, Vasicek O, Nedelcheva P, Blazheva D, Toshkova R, Gardeva E, Yossifova L, Hyrsl P, Vojtek L (2014) Biological activities of selected polyphenol-rich fruits related to immunity and gastrointestinal health. Food Chem 157: 37-44.

Denev P, Lojek A, Ciz M, Kratchanova M (2013) Antioxidant activity and polyphenol content of Bulgarian fruits. Bulg J Agric Sci 19: 22-27.

Duckstein SM, Lotter EM, Meyer U, Lindequist U, Stintzing FC (2013) Phenolic constituents from Alchemilla vulgaris L. and Alchemilla mollis (Buser) Rothm. at different dates of harvest. $Z$ Naturforsch $C$ 68: $529-540$.

Fecka I (2009) Qualitative and quantitative determination of hydrolysable tannins and other polyphenols in herbal products from meadowsweet and dog rose found rugosins $\mathrm{A}, \mathrm{B}$ and $\mathrm{E}$ as a new meadowsweet constituents. Phytochem Analysis 20: 177-190.

Gniewosz M, Synowiec A, Krasniewska K, Przyby1 J, Baczek K, Weglarz Z (2014) The antimicrobial activity of pullulan film incorporat- 
ed with meadowsweet flower extracts (Filipendulae ulmariae flos) on postharvest quality of apples. Food Control 37: 351-361.

Gudej J, Tomczyk M (2004) Determination of flavonoids, tannins and ellagic acid in leaves from Rubus L. species. Arch Pharm Res 11: 1114-1119.

Gutierrez J, Barry-Ryan C, Bourke P (2008) The antimicrobial efficacy of plant essential oil combinations and interactions with food ingredients. Int I Food Microbiol 124: 91-97.

Halliwell B, Zhao K, Whiteman M (2000) The gastrointestinal tract: a major site of antioxidant action? Free Radical Res 33: 819-30.

Kanner J, Lapidot T (2001) The stomach as a bioreactor: dietary lipid peroxidation in the gastric fluid and the effects of plant-derived antioxidants. Free Radical Biol Med 31: 1388-1395.

Kenny TP, Shu SA, Moritoki Y, Keen CL, Gershwin ME (2009) Cocoa flavanols and procyanidins can modulate the lipopolysaccharide activation of polymorphonuclear cells in vitro. J Med Food 12: 1-7.

Kikuzaki H, Hisamoto M, Hirose K, Akiyama K, Taniguchi H (2002) Antioxidant properties of ferulic acid and its related compounds. J Agr Food Chem 50: 2161-2168.

Kratchanova M, Denev P, Ciz M, Lojek A, Mihailov A (2010) Evaluation of the antioxidant activity of medicinal plants containing polyphenol compounds. Comparison of two extraction systems. Acta Biochim Pol 57: 229-234.

Krzymińska S, Tańska A, Kaznowski A (2013) Aeromonas spp. induce apoptosis of epithelial cells through an oxidant-dependent activation of the mitochondrial pathway. J Med Microbiol 60: 889-898.

Lee JE, Kim G-S, Park S, Kim Y-H, Kim M-B, Lee WS, Jeong SW, Lee SJ, Jin JS, Shin SC (2014) Determination of chokeberry (Aronia melanocarpa) polyphenol components using liquid chromatographytandem mass spectrometry: Overall contribution to antioxidant activity. Food Chem 146: 1-5.

Lee JP, Li YC, Chen HY, Lin RH, Huang SS, Chen HL, Kuan PC, Liao MF, Chen CJ, Kuan YH (2010) Protective effects of luteolin against lipopolysaccharide-induced acute lung injury involves inhibition of MEK/ERK and PI3K/Akt pathways in neutrophils. Acta Pharm Sinia 31: 831-838.

Martini S, D’Addario C, Colacevich A, Focardi S, Borghini F, Santucci A, Figura N, Rossi C. (2009) Antimicrobial activity against Helicobacter pylori strains and antioxidant properties of blackberry leaves (Rubus ulmifolius) and isolated compounds. Int J Antimicrob Ag 34: 50-59.
Ou B, Hampsch-Woodill M, Flanagan J, Deemer EK, Prior RL, Huang DJ (2002) Novel fluorometric assay for hydroxyl radical prevention capacity using fluorescein as the probe. J Agr Food Chem 50: 2772-2777.

Ou B, Hampsch-Woodill M, Prior RL (2001) Development and validation of an improved oxygen radical absorbance capacity assay using fluorescein as the fluorescence probe. J Agr Food Chem 49: $4619-4626$.

Pagonis C, Tauber AI, Pavlotsky N, Simons ER (1986) Flavonoid impairment of neutrophil response. Biochem Pharmacol 35: 237-245.

Pincemail J, Thirion A, Dupuis M, Braquet P, Drieu K, Deby C (1987) Ginkgo biloba extracts inhibits oxygen species production generated by phorbol myristate acetate stimulated human leukocytes. Experientia 43: 181-184.

Rota MC, Herrera A, Martinez RM, Sotomayor JA, Jordan MJ (2008) Antimicrobial activity and chemical composition of Thymus vulgaris, Thymus aygis and Thymus byemalis essential oils. Food Control 19: 681687.

Selloum L, Reichl S, Muller M, Sebihi L, Arnhold J (2001) Effects of flavonols on the generation of superoxide anion radicals by xanthine oxidase and stimulated neutrophils. Arch Biochem Biophys 395: 49-56.

Shmid EJ, Gorris LGM (2007) Natural antimicrobials in food preservation. In Handbook of food preservation. Rahman MS, ed. CRC Press, Boca Raton.

Singleton V, Rossi J (1965) Colorimetry of total phenolic with phosphomolibdiphosphotungstic acid reagents. Am J Enol Vitic 16: 144 158.

Smolyakova IM, Andreeva VY, Kalinkina GI, Avdeenko SN, Shchetinin PP (2012) Development of extraction techniques and standardization methods for a common lady's mantle (Alchemilla vulgaris) extract. Pharm Chem J-USSR 45: 675-678.

Suzuki H, Nishizawa T, Tsugawa H, Mogami S, Hibi T (2012) Roles of oxidative stress in stomach disorders. J Clin Biochem Nutr 50: 35-39.

Wang JP, Chang LC, Hsu MF, Chen SC, Kuo SC (2002) Inhibition of formyl-methionyl-leucyl-phenylalaninestimulated respiratory burst by cirsimaritin involves inhibition of phospholipase D signaling in rat neutrophils. N-S Arch Pharmacol 366: 307-314.

Zielinska M, Kostrzewa A, Ignatowicz E (2000) Antioxidative activity of flavonoids in stimulated human neutrophils. Folia Histochem Cyto 38: 25-30. 